\title{
RELAÇÃO ANATÔMICA DO NERVO SUPRAESCAPULAR COM O PROCESSO CORACOIDE, ARTICULAÇÃO ACROMIOCLAVICULAR E ACRÔMIO
}

\author{
ANATOMICAL RELATIONSHIP OF THE SUPRASCAPULAR NERVE \\ TO THE CORACOID PROCESS, ACROMIOCLAVICULAR JOINT AND ACROMION
}

Bernardo Barcellos Terra', Eric Figueiredo Gaspar², Karina Levy Siqueira², Nivaldo Souza Cardozo Filho², Gustavo Cará Monteiro ${ }^{3}$, Carlos Vicente Andreoli ${ }^{3}$, Benno Ejnisman ${ }^{4}$

\section{RESUMO}

Objetivo: Estabelecer a relação anatômica do nervo supraescapular (NSE) localizado na fossa supraescapular com a borda medial da base do coracoide, face articular acromial da articulação acromioclavicular e a borda anterolateral do acrômio. Métodos: Foram dissecados 16 ombros de 16 cadáveres, sendo nove masculinos e sete femininos, mensurando com auxílio do paquímetro a distância do nervo supraescapular (na sua passagem sob o ligamento transverso) com pontos fixos determinados na borda medial da base do processo coracoide, na face articular do acrômio da articulação acromioclavicular e na borda anterolateral do acrômio, correlacionando com a idade e o sexo. Foram excluídos cadáveres com intervenção cirúrgica prévia. Resultados: Com relação à medida do nervo supraescapular, na sua fossa à borda medial da base do processo coracoide, obtivemos uma média de $3,9 \mathrm{~cm}$ (variando de $3,1 \mathrm{~cm}$ a 5,2cm); com relação à articulação acromioclavicular, a média foi de 4,7 (3,9cm a 5,2cm); e, com relação à borda anterolateral do acrômio, a média foi de $6,1 \mathrm{~cm}(5,7 \mathrm{~cm}$ a $6,8 \mathrm{~cm})$. Conclusão: É fundamental o conhecimento exato da anatomia dos nervos da região anterior do ombro para evitar lesões iatrogênicas e para conseguir resultados satisfatórios no tratamento cirúrgico das doenças do ombro, seja ele realizado de forma aberta ou artroscópica.

Descritores - Ombro; Plexo braquial; Anatomia

\section{ABSTRACT}

Objective: To establish the anatomic relationship of the suprascapular nerve (SSN) located in the suprascapular notch to the medial border of the base of the coracoid process, the medial acromial surface of the acromioclavicular joint and the anterolateral edge of the acromion. Methods: We dissected 16 shoulders of 16 cadavers, 9 males and 7 females. The distance from the suprascapular nerve (in its course beneath the transverse ligament) to certain fixed points in the medial base of the coracoid process was measured with the aid of a caliper, as well as to the articular surface of the acromion and the acromioclavicular joint on the anterolateral edge of the acromion. These measures were correlated with age and sex. The specimens with signs of previous surgery were excluded. Results: Measuring the suprascapular nerve in its notch to the medial border of the coracoid process, the base of the coracoid process averaged $3.9 \mathrm{~cm}$ (ranging from $3.1 \mathrm{~cm}$ to $5.2 \mathrm{~cm}$ ), the acromioclavicular joint averaged $4.7 \mathrm{~cm}$ (ranging from $3.9 \mathrm{~cm}$ to 5.2 $\mathrm{cm}$,) and the anterolateral border of the acromion averaged $6.1 \mathrm{~cm}$ (ranging from $5.7 \mathrm{~cm}$ to $6.8 \mathrm{~cm}$ ). Conclusion: Accurate knowledge of the anatomy of nerves of the scapulohumeral region is essential in order to avoid iatrogenic injuries and to achieve satisfactory results in surgical treatment (open or arthroscopic) of shoulder pathologies.

Keywords - Shoulder; Brachial plexus; Anatomy

\footnotetext{
1 - Médico Residente do terceiro ano do Departamento de Ortopedia e Traumatologia da Universidade Federal de São Paulo.

2 - Médico Ortopedista do Centro de Traumatologia do Esporte (Cete/Unifesp) e Membro da Sociedade Brasileira de Ortopedia e Traumatologia.

3 - Médico Assistente do Centro de Traumatologia do Esporte (Cete/Unifesp).

4 - Doutor em Ortopedia e Chefe do Grupo de Ombro do Centro de Traumatologia do Esporte (Cete/Unifesp).
}

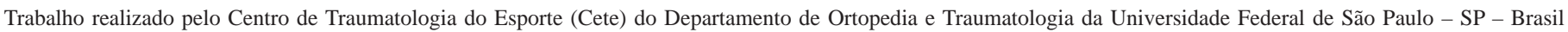
(DOT-Unifesp/EPM).

Correspondência: Rua Borges Lagoa, 783 - 5º andar - Vila Clementino - 04038-032 - São Paulo, SP - E-mail: bernardomed@hotmail.com 


\section{INTRODUÇÃO}

O nervo supraescapular (NSE) é formado basicamente pelas raízes nervosas de $\mathrm{C} 6$ e C7, sendo um ramo do tronco superior do plexo braquial ${ }^{(1)}$. É responsável por $70 \%$ da inervação sensitiva da articulação do ombro, inervando também os músculos supraespinhal e infraespinhal, componentes do manguito rotador ${ }^{(2-4)}$.

Embora seja raro o relato da lesão do nervo supraescapular, o nervo fica em risco de ser lesionado em procedimentos artroscópicos ${ }^{(5-7)}$. A literatura relata que o nervo supraescapular pode ser lesado em procedimentos cirúrgicos que necessitem de uma dissecção posterior da articulação do ombro, assim como avanços do manguito rotador, capsulorrafias, osteotomias para instabilidades posteriores, sutura transglenoide para instabilidade anterior, entre outros ${ }^{(2)}$. Além disso, descreve também mecanismos de compressão e tração deste nervo em atletas ${ }^{(4)}$, bem como uma real possibilidade de ser lesionado durante a realização dos acessos anteriores para abordagem de lesões da articulação acromioclavicular ou fraturas do processo coracoide ${ }^{(3)}$.

Há escassez de estudos relacionando o nervo supraescapular aos parâmetros anatômicos. Dada a importância de se conhecer parâmetros macroscópicos e artroscópicos de referência para este nervo para a realização do tratamento de patologias do próprio nervo ou do ombro, seja procedimentos cirúrgicos abertos ou artroscópicos do ombro, o propósito deste estudo foi mensurar as distâncias entre pontos anatômicos fixos (borda medial da base do processo coracoide, borda anterolateral do acrômio e face articular acromial da articulação acromioclavicular) e o nervo supraescapular em sua fossa sob o ligamento transverso escapular.

\section{MÉTODOS}

Esta pesquisa foi aprovada pela disciplina de anatomia descritiva e topográfica e submetido à avaliação do comitê de ética médica em pesquisa da Universidade Federal de São Paulo - Escola Paulista de Medicina.

\section{Material}

Dissecções foram feitas em 16 ombros de 16 cadáveres, sendo escolhido o lado direito ou esquerdo de forma aleatória, totalizando nove do sexo masculino e sete do sexo feminino, com idade variando entre 46 a 82 anos (média de 59 anos).

As dissecções foram realizadas em cadáveres do Laboratório de Anatomia da Universidade Federal de São Paulo, os quais estavam conservados em solução contendo formaldeído a $10 \%$ por um período entre três e cinco anos.

\section{Método}

As dissecções foram realizadas utilizando a incisão tipo "golpe de sabre”, tendo como parâmetros ósseos o processo coracoide, articulação acromioclavicular e terço médio da clavícula.

Após a incisão, atravessamos a articulação acromioclavicular e rebatemos a clavícula através da desinserção dos ligamentos coracoclaviculares e músculo trapézio. Em seguida, desinserimos o músculo supraespinhal para melhor visualização da incisura escapular na qual encontramos o ligamento transverso superior da escapula e o nervo sob o ligamento (Figura 1).

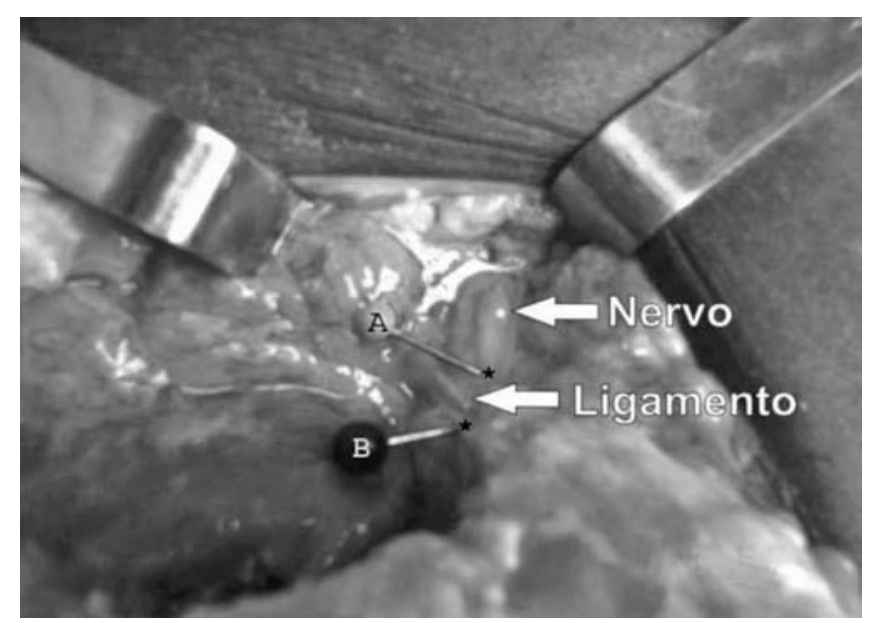

Figura 1 - O nervo supraescapular (alfinete A) e o ligamento transverso superior da escápula (alfinete $B$ )

Ao isolarmos seus ramos, motores para o supraespinhal e infraespinhal e sensitivo para a região posterior da cápsula, mensuramos as distâncias que foram avaliadas no estudo.

As mensurações foram realizadas com o uso de um paquímetro devidamente calibrado. As distâncias foram medidas desde o NSE, em sua fossa sob o ligamento transverso da escápula, até os seguintes marcos anatômicos: borda medial da base do processo coracoide, face articular acromial da articulação acromioclavicular e borda anterolateral do acrômio (Figura 2).

Sendo assim, foram obtidas as seguintes distâncias: A - nervo supraescapular à borda medial da base do processo coracoide; B - NSE à borda anterolateral do acrômio; e C - NSE à face articular acromial da articulação acromioclavicular (Figura 3).

\section{RESULTADOS}

As medidas anatômicas foram: distância A - do NSE na fossa supraescapular à borda medial da base 


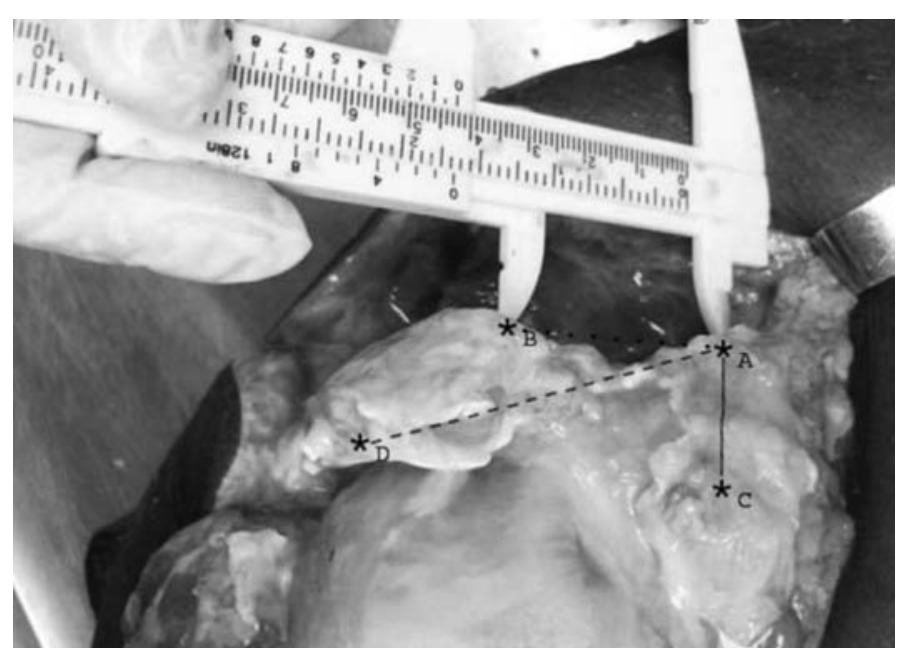

Figura 2 - Distância AB (NSE* à articulação acromioclavicular); distância AC (NSE* à borda medial da base do processo coracoide); distância AD (NSE* à borda anterolateral do acrômio). *NSE: nervo supraescapular

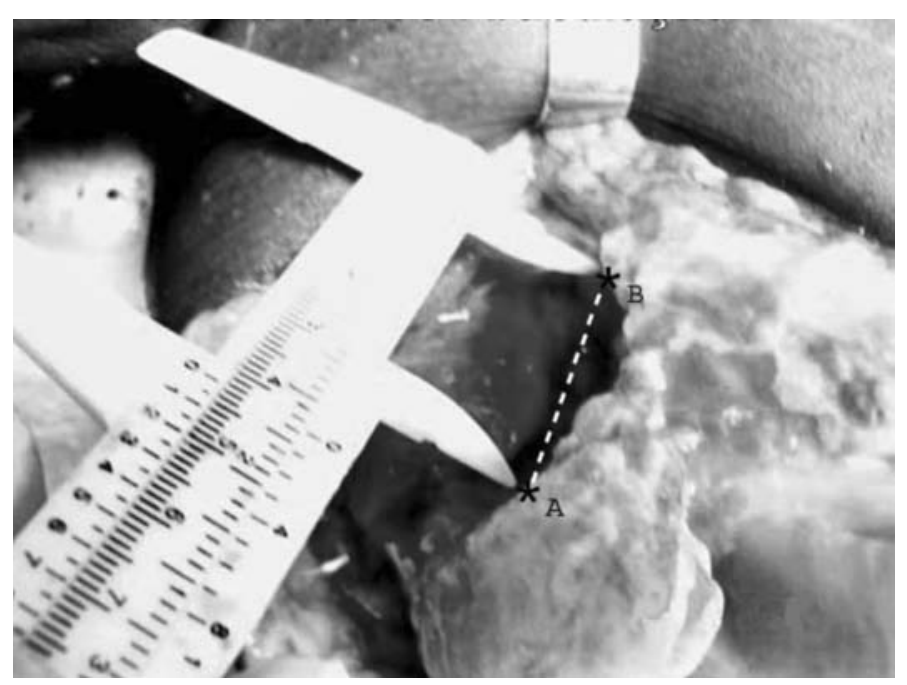

Figura 3 - Distância AB: NSE* à articulação acromioclavicular (face articular do acrômio). *NSE: nervo supraescapular

do processo coracoide: média de 3,9cm; distância B - do NSE na incisura supraescapular à borda anterolateral do acrômio: média de $6,1 \mathrm{~cm}$; distância $\mathrm{C}$ - do NSE à articulação acromioclavicular: média de 4,7cm (Tabelas 1 e 2).

\section{DISCUSSÃO}

A paralisia do nervo supraescapular foi reportada como sendo complicação de procedimentos cirúrgicos nos avanços e no manejo do manguito rotador, capsulorrafias $^{(8-12)}$ e osteotomias da glenoide para as instabilidades posteriores ${ }^{(8,12-14)}$, motivando-nos a estudar os parâmetros anatômicos na região anterior do ombro relacionando-os com o nervo supraescapular.
Tabela 1 - Resultado das medidas em cm das distâncias A, B e $\mathrm{C}$ dos 16 ombros estudados com as médias

\begin{tabular}{|c|c|c|c|c|c|}
\hline N & Sexo & Idade & Distância A & Distância B & Distância C \\
\hline 1 & M & 51 & 3,2 & 5,9 & 3,9 \\
\hline 2 & M & 49 & 3,8 & 5,9 & 4,6 \\
\hline 3 & $\mathrm{~F}$ & 82 & 4,2 & 6,6 & 4,8 \\
\hline 4 & M & 46 & 3,2 & 6,1 & 5,1 \\
\hline 5 & M & 77 & 3,9 & 6,8 & 4,8 \\
\hline 6 & M & 77 & 3,9 & 6,3 & 5,1 \\
\hline 7 & $\mathrm{~F}$ & 71 & 3,6 & 6 & 4,4 \\
\hline 8 & $\mathrm{~F}$ & 63 & 3,3 & 6,4 & 4,1 \\
\hline 9 & M & 47 & 3,1 & 5,7 & 3,9 \\
\hline 10 & $F$ & 57 & 3,2 & 6,3 & 5,1 \\
\hline 11 & M & 55 & 4,4 & 6,3 & 3,9 \\
\hline 12 & M & 53 & 5,2 & 6,1 & 5,2 \\
\hline 13 & $\mathrm{~F}$ & 59 & 5,1 & 6 & 5,1 \\
\hline 14 & $\mathrm{~F}$ & 63 & 3,9 & 5,9 & 5 \\
\hline 15 & $\mathrm{~F}$ & 63 & 3,9 & 5,9 & 5 \\
\hline 16 & M & 48 & 5,2 & 5,9 & 5,2 \\
\hline Média & & 68 & 3,9 & 6,1 & 4,7 \\
\hline
\end{tabular}

Tabela 2 - Média em cm das distâncias A, B e C

\begin{tabular}{l|l}
\hline \multicolumn{1}{c|}{ DISTÂNCIA } & MÉDIA(cm) \\
\hline AB: NSE* à AAC** & $4,7(3,9-5,1)$ \\
\hline AC: NSE* à borda medial da base do PC*** & $3,9(3,1-5,2)$ \\
\hline AD: NSE* à borda anterolateral do acrômio & $6,1(5,7-6,8)$ \\
\hline $\begin{array}{l}{ }^{*} \text { NSE: Nervo supraescapular } \\
\text { *A : Articulação acomioclavicular }\end{array}$ & \\
&
\end{tabular}

Meyer e Graveleau ${ }^{(6)}$ descreveram que o portal lateral transmanguito rotador ficava a uma distância média de 5,3cm deste nervo, devendo-se ter cuidado com o mesmo. Embora seja raro o relato da lesão do nervo supraescapular na literatura ${ }^{(15-17)}$, o nervo fica em risco de ser lesionado em procedimentos artroscópicos ${ }^{(16)}$, principalmente nos portais posteriores e superior (Neviaser) $^{(7,15,18)}$, este último tendo como uma das referências para a sua realização a borda medial do acrômio que, em nosso estudo, apresentou distância média de $4,7 \mathrm{~cm}$ em relação ao nervo.

Nossa pesquisa definiu as distâncias de estruturas anatômicas pré-definidas (borda medial da base do processo coracoide, face articular acromial da articulação acromioclavicular e a borda anterolateral do acrômio) 
com o nervo supraescapular localizado na incisura escapular. No trabalho, obtivemos distância média de 4,7cm e de 3,9 do nervo supraescapular à articulação AC e à borda medial do processo coracoide, respectivamente.

Bigliani ${ }^{(5)}$ mostrou distância média de $1,8 \mathrm{~cm}$ e 2,5cm do nervo supraescapular ao labrum posterosuperior e ao tubérculo supraglenoidal, respectivamente. No presente trabalho, a distância média do nervo à borda anterolateral do acrômio foi de $6,1 \mathrm{~cm}$.

Há escassez de estudos na literatura avaliando a distância do nervo supraescapular ao processo coracoide ${ }^{(19-21)}$. Neste trabalho fizemos a aferição da distância entre a borda medial da base do processo coracoide e o nervo em sua incisura e encontramos distância média de 3,9cm. Este achado é de extrema importância para as técnicas de cirurgia que utilizam o processo coracoide como enxerto, em que a localização do nervo supraesca-

\section{REFERÊNCIAS}

1. Vorster W. The sensory branch distribution of the suprascapular nerve: an anatomic study. J Shoulder Elbow Surg. 2008;17(3):500-2.

2. Shishido $\mathrm{H}$. Injury of the suprascapular nerve in shoulder surgery: an anatomic study. J Shoulder Elbow Surg. 2001;10(4):372-6.

3. Ide J. Does the inferior transverse scapular ligament cause distal suprascapular nerve entrapment? An anatomic and morphologic study. J Shoulder Elbow Surg. 2003;12(3):253-5.

4. Ringel SP, Treihaft M, Carry M, Fisher R, Jacobs P. Suprascapular neuropathy in pitchers. Am J Sports Med. 1978;18(1):80-6.

5. Bigliani LU. An anatomical study of the suprascapular nerve. Arthroscopy. 1990;6(4):301-5.

6. Meyer M, Graveleau N. Anatomic risks of shoulder arthroscopy portals: anatomic cadaveric study of 12 portals. Arthroscopy. 2007;23(5):529-36.

7. Neviaser TJ. Arthroscopy of the shoulder. Orthop Clin North Am. Orthop Clin North Am. 1987;18(3):361-72.

8. Kaspi A. Entrapment of the distal suprascapular nerve. An anatomical study. Int Orthop. 1988;12(4):273-5.

9. Greiner A. The course of the suprascapular nerve in the supraspinatus fossa and its vulnerability in muscle advancement. J Shoulder Elbow Surg. 2003;12(3):256-9.

10. Warner JJP, Krushell RJ, Masquelet A, Gerber C. Anatomy and relationships of the suprascapular nerve: anatomical constraints to mobilization of the Supraspinatus and infraspinatus muscles in the management of massive rotator-cuff tears. J Bone Joint Surg Am. 1992;74(1):36-45.

11. Goldberg BJ, Nirschl RP, McConnell JP, Pettrone FA. Arthroscopic. Transglenoid suture capsulolabral repairs: preliminary results. Am J Sports Med. 1993;21(5):656-65.

12. Yoneda M, Hayashida K, Izawa K, Shimada K, Shino K. A simple and secure pular não é tão estudada e o nervo localiza-se em área de risco, principalmente na região medial e posterosuperior da base do processo coracoide.

\section{CONCLUSÃO}

O presente estudo mostrou as distâncias do nervo supraescapular à borda medial da base do processo coracoide, articulação acromioclavicular e à borda anterolateral do acrômio, medidas estas importantes para o manejo terapêutico das patologias do ombro, evitando colocar em risco o nervo em procedimentos que envolvam a região anterior do ombro, como fixação e redução das fraturas da clavícula, processo coracoide, luxações acromioclaviculares e cirurgias para correção de instabilidades anteriores, bem como na realização das vias cirúrgicas e portais artroscópicos. anchoring system for Caspari's transglenoid multiple suture technique using a biodegradable poly-I-lactic acid button. Arthroscopy. 1996;12(3):293-9.

13. Caspari RB. Arthroscopic reconstruction for anterior shoulder instability. Tech Orthop. 1988;3(1):59-66.

14. Mologne TS, Lapoint JM, Morin WD, Zilberfarb J, O'Brien TJ. Arthroscopic anterior labral reconstruction using a transglenoid suture technique. Results in active-duty military patients. Am J Sports Med. 1996;24(3):268-74.

15. Tibone JE. Posterior capsulorrhaphy for posterior shoulder subluxation. Tech Orthop. 1989;3(4):46-50.

16. Rodeo SA, Forster RA, Weiland AJ. Neurological complications due to arthroscopy. J Bone Joint Surg Am. 1993;75(6):917-26.

17. Small NC. Complications in arthroscopic surgery performed by experienced arthroscopists. Arthroscopy. 1988;4(3):215-21.

18. Matthews LS, Zarins B. Anterior portal selection for shoulder arthroscopy. Arthroscopy. 1985;1(1):33-9.

19. Carson WG Jr. Arthroscopy of the shoulder: anatomy and technique. Orthop Rev. 1992;21(2):143-53.

20. Lo IK, Lind CC, Burkhart SS. Glenohumeral arthroscopy portals established using an outside-in technique: neurovascular anatomy at risk. Arthroscopy. 2004;20(6):596-602.

21. Nassar JA, Wirth MA, Burkhart SS, Schenck RC Jr. Morphology of the axillary nerve in an anteroinferior shoulder arthroscopy portal. Arthroscopy. 1997;13(5):600-5.

22. Pan WJ, Teo YS, Chang HC, Chong KC, Karim SA. The relationship of the lateral cord of the brachial plexus to the coracoid process during arthroscopic coracoid surgery: a dynamic cadaveric study. Am J Sports Med. 2008;36(10):1998-2001.

23. Lo IK, Burkhart SS, Parten PM. Surgery about the coracoid: neurovascular structures at risk. Arthroscopy. 2004;20(6):591-5. 